\title{
Fuzzy Smoothed Composition of Local Mapping Transformations for Non-rigid Image Registration
}

\author{
Edoardo Ardizzone, Roberto Gallea, Orazio Gambino, and Roberto Pirrone \\ Università degli studi di Palermo \\ DINFO - Dipartimento di Ingegneria Informatica \\ Viale delle Scienze - Ed.6 - $3^{\circ}$ piano - 90128 Palermo (Italy) \\ \{ardizzon, robertogallea, gambino, pirrone\}@unipa.it
}

\begin{abstract}
This paper presents a novel method for medical image registration. The global transformation is obtained by composing affine transformations, which are recovered locally from given landmarks. Transformations of adjacent regions are smoothed to avoid blocking artifacts, so that a unique continuous and differentiable global function is obtained. Such composition is operated using a technique derived from fuzzy C-means clustering. The method was successfully tested on several datasets; results, both qualitative and quantitative, are shown. Comparisons with other methods are reported. Final considerations on the efficiency of the technique are explained.
\end{abstract}

Keywords: free form deformation, image registration, fuzzy clustering, function interpolation.

\section{Introduction}

Image Registration is a recent research field in medical imaging with a rapid evolution in the latest years. Indeed, it is a very important task for many applications such as image fusion to integrate data acquired with different modalities (for example MR and PET), therapy evaluation by observing the changes in two images acquired in different times, segmentation purposes, and so on. The problem consists in applying spatial transformations to an input image so that it can be superimposed to a target one. At the end of the automatic process, corresponding pixels will be in the same positions on both the images.

Feature-based approaches recover the global transformation using a sparse set of data such as points or lines that have a correspondence in the two images. The information about the relative positions of these elements allows the registration method to interpolate a global transformation function.

A well known landmark based method used in literature is the Thin-Plate Spline (TPS) surface fitting [1] along with its variants, such as [2, 3], 4] and [5].

This method models a surface constrained to contain several node points. This is done by decomposing the whole transformation in a linear (affine) part, and the superposition of the so-called principal warps that are mutually independent.

P. Foggia, C. Sansone, and M. Vento (Eds.): ICIAP 2009, LNCS 5716, pp. 777 786, 2009.

(C) Springer-Verlag Berlin Heidelberg 2009 
This paper proposes a novel landmark-based registration method. The presented procedure computes independently several local deformations, which are composed subsequently in a global transformation function, which exhibits continuity and smoothness properties. The algorithm is based on concepts derived from Fuzzy C-means clustering [6]. Even though C-means relies on the determination of the clustering centroids, thus requiring an iterative minimization procedure, here the centroids are given as a starting condition, so just membership functions need to be computed. As a result, the optimization step is removed allowing the recovering of the global displacement function composition to be simply the result of quick and efficient sum and product operations.

The landmark-based approach was chosen for several reasons. Firstly, the system is intended just to assist the expert, keeping its role fundamental for the diagnosis process; in addition it allows a fast single-pass computation. Lastly, in our approach, since each local transformation is independent from the others, it is possible to achieve additional speedups by exploiting parallel elaborations.

The paper is arranged as follows: in sect. 2 some thoretical remarks required for the understanding of the work are given. In sect. 3, the registration algorithm steps are presented. Sect. 4 shows the tests performed to validate the algorithm and describes the results, comparing them with the TPS approach. Sect. 4 contains considerations on the algorithm performance and the evaluation of the obtained results. Sect. 5 discusses how the performance can be improved and states the roadmap for further development of the method.

\section{Theoretical Remarks}

Before proceeding with the description of the proposed registration algorithm, some remarks about Fuzzy c-means clustering technique and Thin Plate Spline are required. The former is used for determining a weighting function for composing local transformations, while the latter is a well-known literature method used for landmark-based registration and it is used in this paper for comparative purposes.

\subsection{Fuzzy C-Means}

Fuzzy C-means is a powerful and efficient data clustering method. Each data sample, represented by some feature values, is associated to the clusters of the feature space using some membership degree. Each cluster is identified by its centroid, a point where the feature values are representative for its own class. The original algorithm is based on the minimization of the following objective function:

$$
J_{s}=\sum_{j=1}^{m} \sum_{i=1}^{k}\left(u_{i j}\right)^{s} d\left(\mathbf{x}_{\mathbf{i}}, \mathbf{c}_{\mathbf{j}}\right)^{2}, \quad 1 \leq s \leq \infty
$$

where $d\left(\mathbf{x}_{\mathbf{i}}, \mathbf{c}_{\mathbf{j}}\right)$ is a distance function between each observation vector $\mathbf{x}_{\mathbf{i}}$ and the cluster centroid $\mathbf{c}_{\mathbf{j}}, m$ is the number of clusters, which should be chosen a priori, 


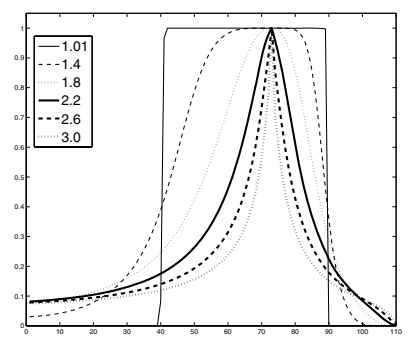

Fig. 1. Example of 1-d membership function plot for different values of $s$

$k$ is the number of observations, $u_{i j}$ is the membership degree of the sample $\mathbf{x}_{\mathbf{i}}$ belonging to cluster centroid $\mathbf{c}_{\mathbf{j}}$ and $s>1$ is a parameter which determines the amount of clustering fuzziness, i.e. the form of membership function Fig. (1), having a value that for common task generally lies in the interval around 2. An additional constraint is that the membership degrees should be positive and structured such that $u_{i, 1}+u_{i, 2}+\ldots+u_{i, m}=1$. The method proceeds as an iterative procedure where, given the membership matrix $\mathbf{U}=\left[u_{i j}\right]$ of size $k$ by $m$, the new positions of the centroids are updated as:

$$
\mathbf{c}_{\mathbf{j}}=\frac{\sum_{i=1}^{k}\left(u_{i j}\right)^{s} \mathbf{x}_{\mathbf{i}}}{\sum_{i=1}^{k}\left(u_{i j}\right)^{s}}
$$

The algorithm ends after a fixed number of iterations or when the improvement of each iteration is substantially small and the new membership values are given by the following equation:

$$
u_{i j}=\frac{1}{\sum_{l=1}^{m}\left(\frac{d\left(\mathbf{x}_{\mathbf{i}}, \mathbf{c}_{\mathbf{j}}\right)}{d\left(\mathbf{x}_{\mathbf{i}}, \mathbf{c}_{\mathbf{l}}\right)}\right)^{\frac{2}{s-1}}}
$$

\subsection{Thin Plate Spline}

One of the classical approaches to image registration is the Thin Plate Spline (TPS). The name is derived from the physical analogy, which involves the bending of a thin metal sheet. In the context of spatial coordinates transformation and image registration, lifting the plate corresponds to displace the image in one direction (i.e. $x, y$ or $z$ axis). The Thin Plate Spline is a parametric interpolation function which is defined by $D(K+3)$ parameters, where $D$ is the number of spatial dimensions of the datasets and $K$ is the number of the given landmark points where the displacement values are known. The function is a composition of an affine part, defined by 3 parameters, and $K$ radial basis functions, defined by an equal number of parameters. In $2 d$ its analytic form is defined as:

$$
g(\mathbf{p})=a x+b y+d+\sum_{i=1}^{K} \rho\left(\left\|\mathbf{p}-\mathbf{c}_{\mathbf{i}}\right\|^{2}\right) w_{i} ; \quad \mathbf{p}=\left[\begin{array}{l}
x \\
y
\end{array}\right] ; \quad \mathbf{c}_{\mathbf{i}}=\left[\begin{array}{l}
c_{x} \\
c_{y}
\end{array}\right]
$$


where $\mathbf{p}$ is the input point, $\mathbf{c}_{\mathbf{i}}$ are the landmark points and $\rho(r)=\frac{1}{2} r^{2} \log r^{2}$ is the radial basis function. All of the TPS parameters are computed solving a linear system defined by a closed-form minimization of the bending energy functional. Such functional is given by:

$$
E_{t p s}=\sum_{i=1}^{K}\left\|y_{i}-g\left(\mathbf{p}_{i}\right)\right\|+\lambda \iint\left[\left(\frac{\partial^{2} g}{\partial x^{2}}\right)^{2}+2\left(\frac{\partial^{2} g}{\partial x y}\right)^{2}+\left(\frac{\partial^{2} g}{\partial y^{2}}\right)^{2}\right] d x d y
$$

The functional is composed by two terms: the data term and the regularization term. The former minimizes the difference between known and recovered displacements at landmark points, the latter minimizes the bending energy of the recovered function, i.e. maximises its smoothness and it is weighted by the parameter $\lambda$. As mentioned before, for this expression a closed-form analytical solution exists, from which is possible to recover all of the required spline function parameters. The main characteristic of this function is that it exhibits minimum curvature properties.

\section{The Registration Algorithm}

The proposed method takes as inputs the target image, the input one, and two sets of control points with their correspondences. The resulting output is the function which implements the mapping between all the points in the two images. The determination of the mapping function is constrained by the displacement of the control points, which can be chosen manually or automatically. Since the purpose of the method is just to find the transformation that achieves the registration, no considerations are reported about control points determination. However, in sect. 5 some clues are given on this topic. The registration procedure is composed of four processing steps (Fig. 22): image space subdivision, local mapping estimation, weighting function computation and global mapping composition. The first step consists in subdividing the whole image into separate regions. For this purpose the adopted strategy is the classic Delaunay triangulation [7] of the given control points, which is optimal in the sense it maximizes all the angles in the generated triangles. With a proper choice of the landmarks, Delaunay triangulation avoids the generation of very thin triangular regions whose vertices are almost collinear, this will produce numerical instability when obtaining local transformations. An example of the output of this procedure is shown in Fig. 3. Once the triangular regions resulting from Delaunay algorithm

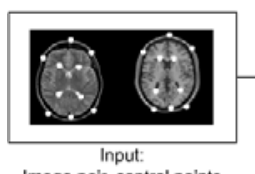

Image pair, control points

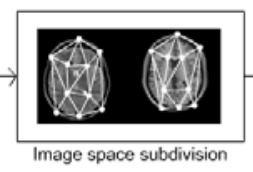

mage space subdivision

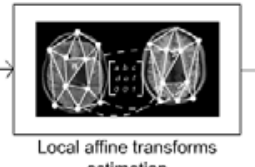

estimation

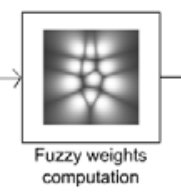

computation

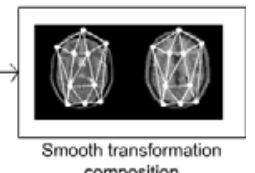

composition

Fig. 2. System block diagram 


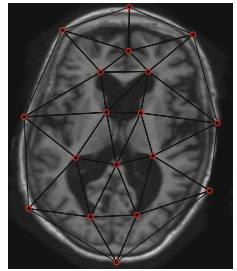

(a)

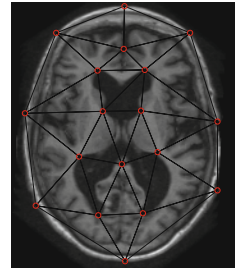

(b)

Fig. 3. From left to right: Delaunay triangulation for input image (a), and target image (b)

are available, the affine transform which best maps each region into the other is recovered by solving the linear system defined by the constraints over the coordinates of the three triangle vertices (6):

$$
\left[\begin{array}{l}
x \\
y \\
1
\end{array}\right]=\left[\begin{array}{lll}
a & b & c \\
d & e & f \\
0 & 0 & 1
\end{array}\right]\left[\begin{array}{c}
x_{0, n} \\
y_{0, n} \\
1
\end{array}\right]=\left[\begin{array}{c}
a x_{0}+b y_{0}+c \\
d x_{0}+e y_{0}+f \\
1
\end{array}\right] \Rightarrow\left\{\begin{array}{l}
x_{1}=a_{1} x_{0,1}+b_{1} y_{0,1}+c_{1} \\
y_{1}=d_{1} x_{0,1}+e_{1} y_{0,1}+f_{1} \\
x_{2}=a_{2} x_{0,2}+b_{2} y_{0,2}+c_{2} \\
y_{2}=d_{2} x_{0,2}+e_{2} y_{0,2}+f_{2} \\
x_{3}=a_{3} x_{0,3}+b_{3} y_{0,3}+c_{3} \\
y_{3}=d_{3} x_{0,3}+e_{3} y_{0,3}+f_{3}
\end{array}\right.
$$

Direct composition of such transformations produces a triangle mesh, and, even though it is spatially congruent and the final global transformation function is continuous, it does not provide good results, because in correspondence of the triangles' boundaries it obviously exhibits crisp edges (Fig. 4a). The surfaces displayed in Fig. 4 represents the resulting displacement along the horizontal direction of the pixel in position $(i, j)$ when it is transformed from the input image to the target one, respectively using direct composition (a), the proposed method (b), and TPS (c). The global transformation is thus completely defined by a couple of surfaces, one for each dimension. We need a further processing in order to make the global transform function smooth. To achieve this purpose, we weigh the local mapping functions in a proper way, using concepts related to Fuzzy c-means clustering technique. In sec. 2.1 such technique is recalled in more details. Even though centroids and fuzzy membership concepts are useful for our purposes, no clustering process is required, and no minimization is performed actually. If we assume the center of mass of each triangle as a cluster centroid in the feature space defined by the pixel spatial coordinates $(i, j)$ (in [8] the landmarks coordinates are roughly used for this purpose), then membership values relative to each cluster can be assigned to each pixel in the image. Membership values will be more or less strong according to its displacement w.r.t. each centroid. Due to the summation constraint, it is possible to use membership values as weighting terms for the local mapping functions. The amount of decay related to distance variation, and consequently the membership value itslef, is controlled by means of the $s$ exponent, which is the unique tunable parameter in this registration scheme (see sec. 4 for further details). 


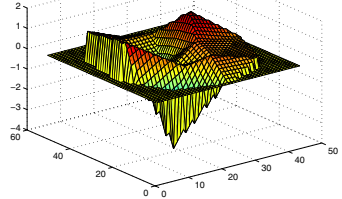

(a)

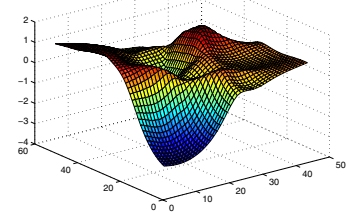

(b)

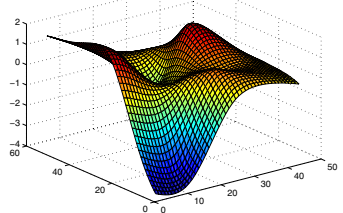

(c)

Fig. 4. From left to right: an example of the resulting mapping function with affine direct composition (a), fuzzy smoothed composition (b), TPS surface fitting (c)

Given the above conditions, the new coordinate vector $\mathbf{v}_{\mathbf{i j}}$ of the pixel at position $(i, j)$ is given by:

$$
\mathbf{v}_{\mathbf{i j}}=\sum_{n} u_{i j, l}\left(\mathbf{T}_{\mathbf{n}} \cdot \mathbf{x}_{\mathbf{i j}}\right)
$$

where $u_{i j, l}$ is the membership degree of the pixel of coordinates $\mathbf{x}_{\mathbf{i j}}$ for the $l$-th centroid while $\mathbf{T}_{\mathbf{n}}$ is the affine transformation for the $l$-th centroid, recovered in the previous step.

This results in a smooh transformation function with no discontinuities, and governable smoothness by adjusting the $s$ parameter. An example is shown in Fig. 4b. For comparisons purposes, in Fig. 4k, the transformation obtained from TPS approximation using the same inputs is also shown.

Another remarkable advantage of our approach is that the time-consuming minimization procedure required to fit a global transformation function is replaced by solving analitically at most $2 n-2-b$ linear systems of three equations, where $n$ is the total number of control points and $b$ is the number of vertices of the convex hull defined by the control points. What needs to be computed is just the membership matrix $\mathbf{U}$ using (7). The metric used to compute the distance function is the Euclidean distance because it is sufficient to take into account just spatial closeness between pixels and control points. Such method can be used both for 2- $D$ images and $3-D$ volumes. The only difference is in the number of transformation functions to be computed that is one for each dimension.

\section{Results and Discussion}

The proposed method has been implemented and tested on sets of both real (100 sample pictures) and synthetic medical images provided by Brainweb 9 10 1112 (50 sample pictures). Some preliminary tests were conducted on a template pattern. The image contains a white circle on black background, Fig. 57. The image was strongly deformed as in Fig. 5b. Then the registration procedure was applied using a different number of landmarks both with our algorithm, and the TPS one. The results are shown in Fig. 51 and Fig. 5d. Differences between the two methods are shown in Fig. [5e, where the dark contour represents the TPS approximation scheme, while the bright one refers to the proposed method. 


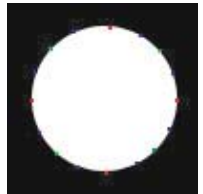

(a)

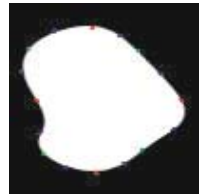

(b)

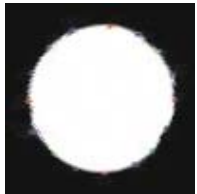

(c)

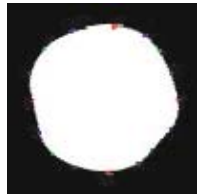

(d)

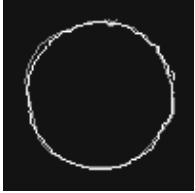

(e)

Fig. 5. From left to right: results on a template image using 18 control points. Base image (a), input image (b), registered image using our method (c), and using TPS approach (d), difference between the two approaches (e) where the bright contour represents our method, while the dark one is related to the TPS approach.

The figure shows that a better image recovery is obtained using the proposed method, though differences are quite subtle, just in correspondence of the area of large deformations, which are better modelled by local transformations. (like in the middle part of the pattern).

The second class of tests consists in the use of synthetic medical images manually deformed and then registered back onto their original version. In this case the performance has been evaluated using several similarity metrics: sum of squared difference $(S S D)$, mean squared error $(M S E)$ and mutual information $(M I)$ as objective measures, and Structural Similarity $(S S I M)$ [13] as a subjective one. The algorithm was run using different values for the $s$ parameter. Visual results for the proposed method applied to a sample image are depicted in Fig. 6, while the measures are summarized in Table 1. Comparisons with TPS approach are also presented. The outcomes point out that the presented method performs better than TPS irrespectively to the similarity measure taken into account.

Other merely visual tests were performed on various photographic images in order to evaluate the performance of the algorithm for other purposes, such as morphing. In Fig. 7 the results obtained morphing two sample images are shown. As results from the tests reported, in the average case comparisons with TPS approach resulted in a better performance of our method. In addition, it results even more efficient and faster: as an example on a P4 machine equipped with MS Windows Vista and Matlab R2008a, the registration of a 208x176 pixels image using 18 landmarks (Fig 3 and Fig[6]), took around $40 \%$ of the time taken by

Table 1. Results summary for different values of $s$ and $\lambda$, best measures underlined

\begin{tabular}{c|cccc|cccccc}
\hline \hline \multicolumn{9}{c|}{ Fuzzy Approximation } & \multicolumn{5}{c}{ TPS Approximation } \\
\hline$s$ & MSE & SSD & MI & SSIM & $\lambda$ & MSE & SSD & MI & SSIM \\
1.2 & 29.3167 & 1073225 & 1.6627 & 0.7985 & 0.0001 & 30.8739 & 1359829 & 1.2067 & 0.7918 \\
1.4 & 29.2199 & 1069683 & 1.6649 & 0.8001 & 0.0006 & 30.4943 & 1149111 & 1.2487 & 0.8135 \\
1.6 & 29.0541 & 1063612 & 1.6725 & $\underline{0.8021}$ & 0.0011 & 31.0487 & $\underline{1136312}$ & 1.2502 & $\underline{0.8145}$ \\
1.8 & 29.9451 & 1096229 & 1.6570 & 0.7935 & 0.0016 & 30.2244 & 1168397 & 1.2505 & 0.8142 \\
2.0 & 30.4536 & 1114847 & 1.6488 & 0.7886 & 0.0021 & 29.6988 & 1181151 & 1.2494 & 0.8139 \\
2.2 & 31.2665 & 1144603 & 1.6281 & 0.7716 & 0.0026 & 29.9593 & 1171422 & 1.2501 & 0.8139 \\
2.4 & 31.7396 & 1161925 & 1.6154 & 0.7593 & 0.0031 & 29.2244 & 1165640 & 1.2496 & 0.8137 \\
2.6 & 32.2610 & 1181010 & 1.6022 & 0.7491 & 0.0036 & 30.9593 & 1172249 & 1.2489 & 0.8132 \\
2.8 & 32.6101 & 1193790 & 1.5934 & 0.7413 & 0.0041 & 30.9593 & 1174901 & 1.2485 & 0.8130 \\
3.0 & 32.9059 & 1204621 & 1.5853 & 0.7339 & 0.0046 & 30.2244 & 1168803 & 1.2490 & 0.8095 \\
\hline \hline
\end{tabular}




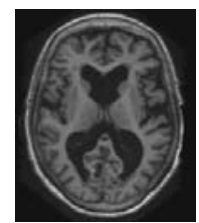

(a)

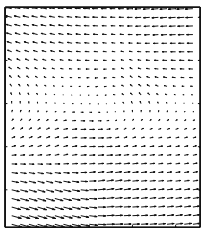

(e)

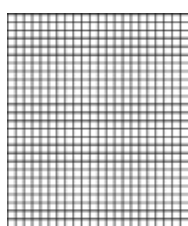

(b)

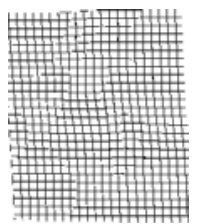

(f)

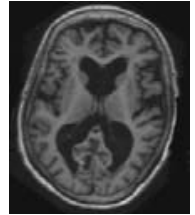

(c)

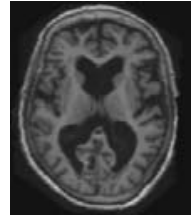

(g)

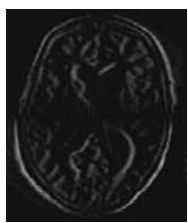

(d)

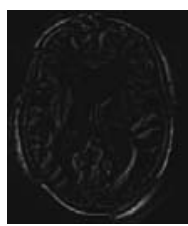

(h)

Fig. 6. From left to right: ground truth (a), initial registration grid (b), deformed version of the image (c), initial error image (d), deformation field (e), deformed registration grid (f), registered version with 18 landmark points (g), final error image (h)

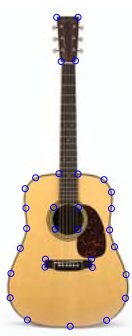

(a)

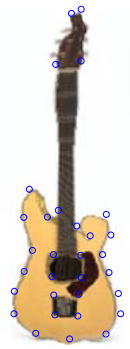

(b)

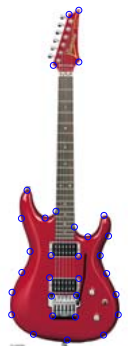

(c)

Fig. 7. From left to right, qualitative results on a photographic image: input image (a), registered image using 31 landmark points (b), target image (c)

TPS approximation (execution times are approximately 14.8 seconds versus 37.2 seconds). Notwithstanding for both methods is required the solution of linear systems, in the case of TPS, the system to solve is as larger as the number of control points. The computational burden of our technique is much smaller, since simple (and parallelizable) systems of just six equations need to be solved. The results have then to be combined with simple distance measures and weighted sums. As regards the tuning of the smoothing parameter $s$, experiments shown that the optimal value generally lies in a range between 1.4 and 2, and a few one-dimensional search attempts (3-4 trials on average) using bisection strategies such as golden ratio, are enough to find the right solution, keeping the method convenient. In addition, similar problems exist in the TPS approach where the regularization parameter $\lambda$ should be determined.

Comparing the size of data structures for the whole spatial transformation, it can be seen that our approach is linear in the number of triangles (which are at 
most $2 M-2-b$, see sec. 3), and as a consequence, of control points. Storing space for affine transformation parameters (six for each triangle) is in the order of $12 M$, where $M$ is the number of control points. Moreover, $2 M$ values, i.e. one for each triangle, for the membership degrees of each point are needed. However, once every single pixel has been transformed, its membership degree can be dropped, so the total data structure is $14 M$ large. TPS approximation has a little more compact structure, because it needs just to maintain the $2(M+3)$ $x$ and $y$ surface coefficients ( $M$ for the non-linear part and 3 for the linear one in each dimension). However, the storing complexity is $O(M)$ in both cases, i.e. linear in the number of control points used, and thus equivalent.

\section{Conclusions and Future Work}

A novel method aimed to free form deformation and registration has been presented. This technique computes the whole global transformation by composing several local transformation. Such composition is kept continuous and smooth by means of applying weighting functions to each pixel, depending on its position. Such weighting term is obtained from fuzzy membership of each pixel to each region in the image. Fuzzy C-means clustering algorithm is used for this purpose. However, since just the concepts of centroids and fuzzy membership degree are used, no clustering operation needs to be performed actually. The performance of the proposed method was evaluated with several tests using both objective and subjective measures, and the results were compared to free form deformation technique based on TPS surface approximation. From this study, it resulted that the presented method provides better results. In addition, it is substantially faster due to its straightforward computation requiring no optimization.

Future work will be oriented towards the use of the same technique for registering 3- $D$ medical volume datasets using parallelization of computations and an automatic feature detection. The feature information are intended to be chosen by means of contour curvature evaluation and similarity considerations on the inner structures. Subsequently, the control points-based approach will be left towards a fully automatic pixel-based elastic registration framework.

\section{References}

1. Bookstein, F.L.: Principal warps: thin-plate splines and the decomposition of deformations. IEEE Transactions on Pattern Analysis and Machine Intelligence 11(6), 567-585 (1989)

2. Arad, N., Dyn, N., Reisfeld, D., Yeshurun, Y.: Image warping by radial basis functions: Application to facial expressions. Computer Vision, Graphics, and Image Processing. Graphical Models and Image Processing 56(2), 161-172 (1994)

3. Rohr, K., Stiehl, H.S., Sprengel, R., Buzug, T.M., Weese, J., Kuhn, M.H.: Landmark-based elastic registration using approximating thin-plate splines. IEEE Transactions on Medical Imaging 20(6), 526-534 (2001) 
4. Bartoli, A., Perriollat, M., Chambon, S.: Generalized thin-plate spline warps. IEEE International Conference on Computer Vision and Pattern Recognition, cvpr (2007)

5. Johnson, H.J., Christensen, G.E.: Consistent landmark and intensity-based image registration. IEEE Transactions on Medical Imaging 21, 450-461 (2002)

6. Bezdek, J.C.: Pattern Recognition with Fuzzy Objective Function Algorithms (Advanced Applications in Pattern Recognition). Springer, Heidelberg (1981)

7. Delaunay, B.N.: Sur la sphère vide. Bulletin of Academy of Sciences of the USSR (6), 793-800 (1934)

8. Ardizzone, E., Gallea, R., Gambino, O., Pirrone, R.: Fuzzy c-means inspired free form deformation technique for registration. In: WILF, International Workshop on Fuzzy Logic and Applications (2009)

9. Cocosco, C.A., Kollokian, V., Kwan, R.K.S., Pike, G.B., Evans, A.C.: Brainweb: Online interface to a 3d mri simulated brain database. NeuroImage 5, 425 (1997)

10. Kwan, R.K.S., Evans, A.C., Pike, G.B.: Mri simulation-based evaluation of imageprocessing and classification methods. IEEE Transactions on Medical Imaging 18(11), 1085-1097 (1999)

11. Kwan, R.K.-S., Evans, A.C., Pike, G.B.: An extensible mri simulator for postprocessing evaluation. In: Höhne, K.H., Kikinis, R. (eds.) VBC 1996. LNCS, vol. 1131, pp. 135-140. Springer, Heidelberg (1996)

12. Collins, D.L., Zijdenbos, A.P., Kollokian, V., Sled, J.G., Kabani, N.J., Holmes, C.J., Evans, A.C.: Design and construction of a realistic digital brain phantom. IEEE Trans. Med. Imaging 17(3), 463-468 (1998)

13. Wang, Z., Bovik, A.C., Sheikh, H.R., Simoncelli, E.P.: Image quality assessment: From error visibility to structural similarity. IEEE Transactions on Image Processing 13, 600-612 (2004) 